\title{
Multiparticle aggregation model for dendritic growth applied to experiments on amorphous Co-P alloys
}

\author{
Angel Sánchez \\ Escuela Politécnica Superior, Universidad Carlos III de Madrid, C. Butarque 15, E-28911 Leganés, Madrid, Spain \\ and Theoretical Division and Center for Nonlinear Studies, Los Alamos National Laboratory, Los Alamos, New Mexico 87545 \\ M. J. Bernal and J. M. Riveiro \\ Departamento de Física Aplicada, Facultad de Ciencias Químicas, Universidad de Castilla-La Mancha, \\ E-13004 Ciudad Real, Spain
}

(Received 28 June 1994)

\begin{abstract}
We introduce a multiparticle biased diffusion limited aggregation model for dendritic growth. Its most relevant feature is that it includes the overall effect of strong applied electric fields and therefore applies to nonequilibrium situations. We compare simulations of a two species version of our model to actual experiments on preparation of amorphous Co-P alloys with very good agreement: The model accurately reproduces the dependence of composition, morphology, and growth time of the alloy on the current. We conclude with a discussion of specific predictions and possible generalizations of the model.
\end{abstract}

PACS number(s): $68.70 .+w, 82.20 . \mathrm{Wt}, 05.40+\mathrm{j}, 61.50 . \mathrm{Cj}$

In the last few years it has become clear from different experiments that the same underlying mechanism governs disparate growth processes like electrochemical deposition (ECD), fluid-fluid displacement, growth of bacterial colonies, or dielectric breakdown, to name a few [1,2]. It has also been established that small variations of the parameters controlling this mechanism, generally known as Laplacian growth, give rise to a rich variety of patterns, ranging from fractal to dendritic, open, or dense ones (see, e.g., the reprints in [2]). Several generalizations of the diffusion limited aggregation [3] and dielectric breakdown models [4] (see also [5] and references therein) have been proposed to account for those patterns with moderate success. In this regard, Erlebacher et al. [6] presented recently simulations of a model that agrees qualitatively well with these experiments. However, no detailed quantitative comparisons between aggregation models and experiments have been carried out as far as we know. It would be very interesting to have such studies to gain insight on the complicated (and theoretically far from understood) issue of nonequilibrium growth.

In this Rapid Communication, we present a multiparticle, biased diffusion limited aggregation (MBDLA) model for far from equilibrium growth phenomena and we apply it to recent experiments on growth of amorphous cobalt-phosphorus alloys by electrochemical deposition [7]. Amorphous Co-P alloys have very many interesting applications and hence have been widely studied in the literature [8]. On the other hand, modeling of amorphous alloy growth has the advantage that no (crystalline) order parameter is needed as in the case of crystal growth. For this reason we believe this particular application of MBDLA can be of interest for many researchers in this and related systems (NiP, NiPB, NiCoP, etc.). However, MBDLA can be straightforwardly used to describe other experimental situations: Thus, for comparison with the Co-P system, MBDLA will include two components, but it can be applied to either one or several component processes as well. To date, to our knowledge, only Nagatani and Sagués [9] have studied a two-component

multiparticle DLA [10] model from the theoretical viewpoint without including comparison with experiments.

To make the paper more self-contained, we briefly summarize the experimental results we try to recover from our MBDLA model. The reader is referred to [7] for a more detailed report. $\mathrm{Co}_{88} \mathrm{P}_{12}$ amorphous alloys for use as soft magnetic materials were produced by dendritic growth on the edge of a $\mathrm{Cu}$ substrate by ECD with high density current. The initial bath composition was $75 \%$ Co and $25 \% \mathrm{P}$ ions; slightly different compositions led essentially to the same results. We note that all these experiments (i.e., all the experimental points plotted in Fig. 1 that we discuss below) were carried out in constant current conditions, rather than constant applied voltage, and a parallel geometry. It was observed that when the current density $J$ was increased the cobalt fraction in the composition [11] of the alloys increased as well, until it saturated at $88 \%$ Co atoms for $J \geqslant 4 \times 10^{4} \mathrm{~A}$

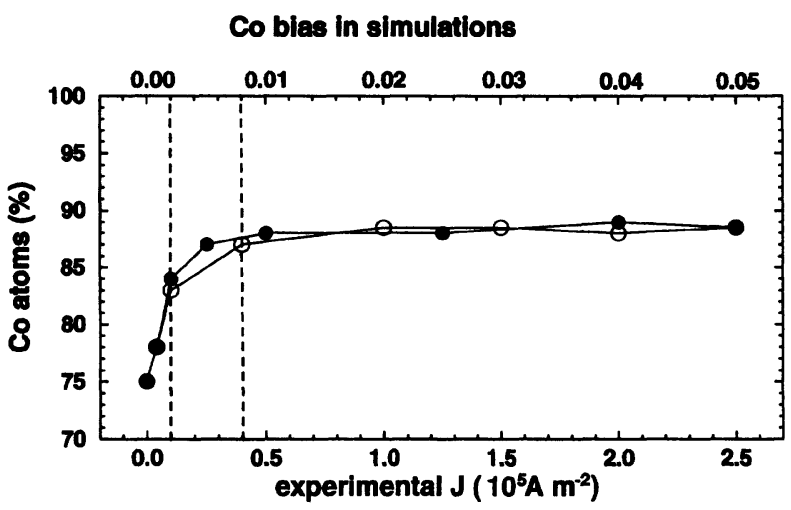

FIG. 1. Alloy composition vs experimental current density $J$ and Co bias $p$ in simulation. Full circles are obtained from simulation and empty ones from experiment. Leftmost experimental and simulated points lie on top of each other. Lines are only a guide to the eye. 


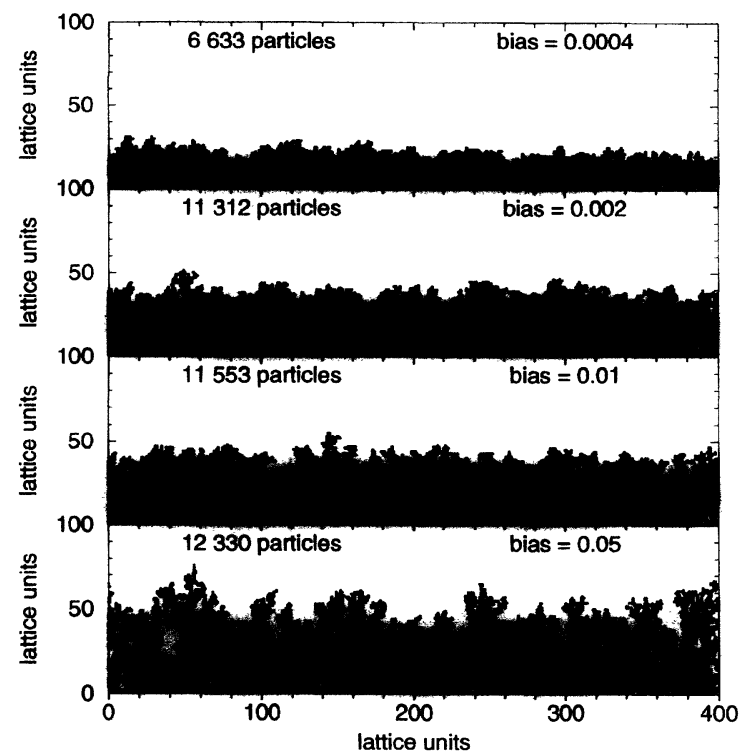

FIG. 2. Clusters obtained for different currents and times. Bottom to top: $J=2.5,0.5,0.1$, and $0.02 \times 10^{5} \mathrm{~A} \mathrm{~m}^{-2}$ (equivalent $p=0.05,0.01,0.002$, and 0.0004 ), whereas $T_{M}=2,10,50$, and 60 min (equivalent $T_{\text {sim }}=2.8,14,70$, and $84 \times 10^{7}$ time steps). Note that the morphology becomes more open when increasing the current, becoming quite planar for the smallest $p$ value. The top aggregate was grown with $s$ four times the one it should have according to the linear relationship, to make it visible here. With the proper $s$ value $T_{M}$ would be $300 \mathrm{~min}$ (equivalent to $350 \times 10^{7}$ time steps).

$\mathrm{m}^{-2}$. Three regions with different types of electrolytic growth were found (see Fig. 1). For small $\left(0 \leqslant J \leqslant 10^{4}\right.$ $\mathrm{A} \mathrm{m}^{-2}$ ) $J$ values, the growth was planar and the Co concentration increased with $J$. Typical grown samples had a size of a few tens of micrometers. Intermediate $J$ values $\left(10^{4}\right.$ A m ${ }^{-2} \leqslant J \leqslant 4 \times 10^{4} \mathrm{~A} \mathrm{~m}^{-2}$ ) gave rise to dendritic morphologies, and again to an increase of Co concentration with $J$. The typical size of the dendrites was in the range of a few milimeters (for a picture, see Fig. 2 in [7]). Finally, for $J \geqslant 4 \times 10^{4} \mathrm{~A} \mathrm{~m}^{-2}$, the growth remained dendritic but the composition reached a constant value independent of $J$. In this regime, deposits were always homogeneous, even if large current density fluctuations occurred. This was checked by preparing samples with different deposit times (from 10 to $60 \mathrm{~min}$ ). Another interesting remark on the alloy morphology is that the larger $J$ is, the more and the greater the empty spaces in the obtained dendritic sample are. Finally, an inverse linear relation between the time $T_{M}$ needed to grow a given mass $M$ of alloy and the current used to prepare it was found, i.e., $T_{M} \sim J^{-1}$.

We now turn to the aggregation model we propose to reproduce this phenomenology, which is that of a twocomponent diffusion (Laplacian) problem with a perturbation term due to the electric field, plus another extra term coming from the interaction between the two species during the deposition process ( induced codeposition, see below, see also [12]). Our algorithm starts from a number of random walkers ("ions") randomly placed on a square lattice, with the same composition with respect to the bath as the experimental one $(\sim 10 \%$ sites occupied $)$, and in the same relative compositional ratio (three times more Co than $\mathrm{P}$ ). Bidimensional lattices were chosen to avoid large computational times while keeping a significant number of walkers. The lattice boundary was rectangular (similar to the experimental one), and we took its lower side to be the cathode. Boundary conditions were periodic in the direction parallel to the cathode and reflective in the perpendicular one (with the possibility to stick to the cathode, of course). Simulations were done on a $300 \times 400$ lattice; different sizes did not modify the results. The initial condition evolves in time according to the following rule. Every time step, a walker is chosen at random (all of them with equal probability), and one of its four nearest neighbors is chosen to be its destination site. This is done according to the following probability: 0.5 to move horizontally, i.e., parallel to the cathode, $0.25+p$ to move vertically downwards (towards the cathode), and $0.25-p$ to move vertically upwards. We hereafter call $p$ the Co bias for reasons that will become clear in the next paragraph. After choosing a site to move to, motion actually takes place if there is no other walker at that precise node. In case the chosen site belongs to the growing aggregate (in the first time step, the lower layer of the lattice), the walker's preceding position is added to the aggregate with probability $s$, otherwise the walker stays there (and can move in future time steps) with probability $1-s$. We term $s$ the sticking probability (often called noise reduction parameter) and again postpone its discussion to the next paragraph. If the walker is added to the aggregate, a new one is created at a random site, of the same nature as the one that has clung to the cluster [13]. This completes a time step, and the definition of the algorithm.

The physics underlying MBDLA is the following. First of all, multiparticle DLA [10] is needed to include the consequences of having a finite concentration of two components simultaneously. Second, we have to introduce the large electric current. This we do by means of $p$, the fundamental parameter of the model. It seems reasonable that the larger the current density in the experiment, the larger $p$ must be in the model to reproduce it. Moreover, $p$ allows one to account for the above mentioned induced codeposition [12] as we describe now. We have two ions, Co (positive) and $\mathrm{P}$ (negative) in the solution, with two forces making them move: diffusion and electric force. In the case of Co, both drag the ion to the cathode. However, in the case of P, the electric force goes towards the anode. Therefore, as $\mathrm{P}$ deposits in the cathode (and it is well known that $\mathrm{P}$ alone would not deposit there), it has to be due to diffusive forces, i.e., the accumulation of $\mathrm{Co}$ ions near the cathode induces electroconvective effects on $\mathrm{P}$ ions. The simplest way to take all these processes into account is to relate the bias for Co walkers to the bias for $\mathrm{P}$ walkers, in the form $p_{\mathrm{Co}}=p, p_{\mathrm{P}}=\alpha p$; in this fashion, Co ions will drag $\mathrm{P}$ ones towards the cathode with a strength proportional to their one attraction to the negative electrode. The $\alpha$ parameter is the adjustable one and will be determined below from the experimental results. The remaining physicochemical complicated processes, difficult to quantify, are included in the parameter $s$. In particular, notice that $s$ is related to the activation energy of the deposition process itself, and that it should be easier for faster ions to deposit. Hence $s$ must certainly be related in some way to the current, i.e., to $p$, and moreover, $s$ should grow with $p$ [14]. 


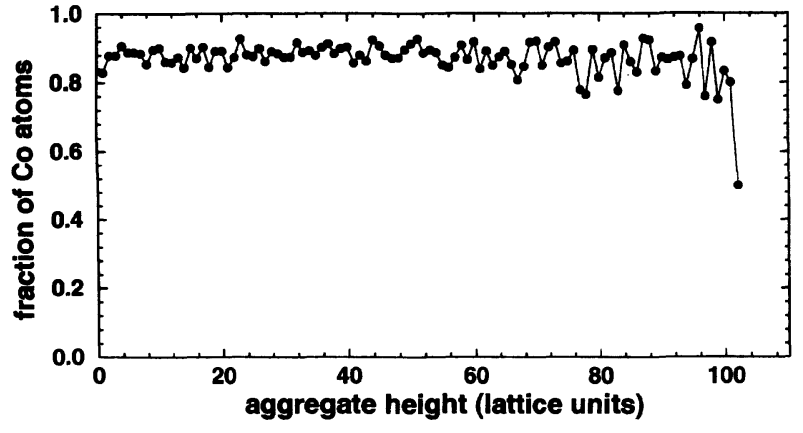

FIG. 3. Alloy composition as a function of the aggregate coordinate perpendicular to the cathode. Fluctuations at high values come from the the fact that there are very few particles near the tips. This aggregate was grown with $J=2 \times 10^{5} \mathrm{~A} \mathrm{~m}^{-2}(p=0.04)$ during $2.5 \mathrm{~min}\left(3.5 \times 10^{7}\right.$ time steps).

We adopted this hypothesis and we consequently did not choose $s$ independently but, rather, established a relationship between both parameters. Since the exact relationship is of course unknown, we took $s$ in the interval $0<s \leqslant 1$ for each value of $p$. We found that the resulting concentration value was rather insensitive to the precise value chosen for $s$ (see further discussion of this parameter below). This is very important, because otherwise the model would not be of much interest as this relation would have to be determined for every particular case. Thus, to summarize, we want to stress that the main model control parameter is $p$ (hence the name MBDLA), and the adjustable one is $\alpha$.

We have performed a detailed simulation program of the above model. For simplicity, we started by fixing $\alpha=0$, i.e., Co ions were biased random walkers whereas $\mathrm{P}$ ones were pure random walkers. Physically, $\alpha=0$ means that concentration gradients (due to induced codeposition) forces are exactly balanced by the electrical force. With this choice, we reproduced the composition saturation and the homogeneity of the samples, but the saturation value of the dendritic alloys was wrong, around $92 \%$ Co atoms. We therefore gave up the (otherwise unrealistic) hypothesis of exact force balance and modified $\alpha$ to get quantitative agreement with the experiments. It turned out that $\alpha=0.3$ gave rise to the same composition of the alloys as in the experiment, $88 \%$ Co atoms. It is important to notice that this implies that $\mathrm{P}$ negative ions move towards the negative electrode, and this can only come from the concentration gradient originated by the induced codeposition influence, as we mentioned above.

The simulation outcome (with $\alpha=0.3$ ) is summarized in Figs. 1-3. Figure 1 plots the dependence of the composition of the alloys on the current density, showing very good agreement between MBDLA and experiments. It is most remarkable that with an adjustable parameter, $\alpha$, we recover the behavior of the concentration in the whole range of currents. An important conclusion that must be drawn from this plot is that there is a direct relationship between the current density in the experiments and the value of $p$. The exact prefactor will depend on the type and the size of the lattice, but for the lattice we are dealing with here, $J=p \times 5 \times 10^{6} \mathrm{~A}$ $\mathrm{m}^{-2}$. After we have determined the $p$ values that correspond to physical $J$ values from the saturation curve, we are not free to modify $p$ anymore. Hence the $p$ values obtained from this relationship must also reproduce the features of the aggregates grown with different $J$ if our model is correct. Besides, we have to fix the relationship between the parameter $s$ and $p$. After trying several functional forms we chose a simple linear dependence $(s \approx 20 p$ in the range $0 \leqslant p \leqslant 0.05$ ) because it already gave a very good agreement with the aggregate morphology. Other dependences we tried were parabolic and hyperbolic tangent, in different ranges of $p$. The obtained morphologies are similar to the linear one, so it is enough to assume a smooth increasing dependence which we thus exemplify by the linear one. These morphologies can be seen in Fig. 2. The aggregates in this figure were obtained in the following form: A number of actual experimental values of $J$ and the corresponding $T_{M}$ were taken. From $J$, the values of $p$ to be used in the simulations were computed through the above relationship. We then simulated the largest of them, $p=0.05$, for a certain number of time steps. This allowed us to obtain an equivalence between simulated and actual time: We took the number of time steps in the simulation to be proportional to the growth time for $J=2.5 \times 10^{5} \mathrm{~A} \mathrm{~m}^{-2}$ (i.e., $p=0.05$ ), which was $2 \mathrm{~min}$. By this procedure, we found that $1 \mathrm{~min}=1.4 \times 10^{7}$ simulation time steps. Finally, we simulated the rest of the cases stopping the simulation at a number of time steps equivalent to the experimental, physical time. The agreement was fully satisfactory, and in all cases a number of particles of the order of 12000 was obtained.

Morphologies are also very similar to the ones arising in the experiment, the ones grown with larger $s$ (which is proportional to $p$ ) being more open as shown in Fig. 2. In the limit when the current $J$ is very close to 0 , masses obtained in actual experiments (by this and other groups) are rather small. This is so because the deposit is not allowed to grow for a long enough time as would be required by the relation $T_{M} \sim J^{-1}$. This is shown in the top plot of Fig. 2, an aggregate grown for the equivalent of $60 \mathrm{~min}$ which would have needed twice that time to reach $\sim 12000$ particles ( $s=0.04$, greater than it should be to induce fast growth). In this small $J$ regime, the morphology of the aggregates becomes less dendritic and more compact, reproducing the change reported in [7] from planar to dendritic shapes upon increasing the current density. The fact that the parameter $s$ is responsible for the change in morphology can be seen by comparing Fig. 2 to the results in the second paper in [10]. We see that the morphologies are very similar in both cases for all values of $s$ (aggregates are compact for small $s$, becoming more open as $s$ increases), in spite of the fact that in our model random walkers are biased. This means that $s$ is the parameter that controls compactness, as in nonbiased multiparticle DLA, whereas $p$ governs composition and growth time. Note also that the associated change in the number of particles is related to the experimental change of scales, from micrometers to millimeters. Finally, Fig. 3 shows an example of the cluster composition as a function of its height. The alloy bulk is fairly homogeneous, and fluctuations arise only at the higher parts, where only the few particles at the growing tips contribute. The same degree of homogeneity was seen for all $J$ (equivalently $p$ ) values considered.

In summary, we have presented a simple model, MBDLA, that reproduces Co-P growth by ECD in conditions very far 
from equilibrium. The main success of the model is that it can be compared quantitatively to experiments, as the parameter $p$ and the simulation time can be straightforwardly correlated to the physical $J$ and growth time. The very good agreement in every aggregate characteristic allows us to conclude that the model captures all the essential physics of the growth process in a very simple way. We stress that this model can be used to interpret other experiments as well as to predict new effects that might arise when changing the experimental conditions. For instance, MBDLA simulations predict that in ECD experiments for low $J$, aggregates develop dendritic shapes for long times (compact dendritic morphology), thus decreasing the quality of the deposit for applications. As regards generalizations, aside from the obvious one to different numbers of species, the model can be adapted to situations where crystalline deposits are obtained by imposing constraints on the sticking probability depend- ing on the type of the neighbors and/or the number of them. Finally, theoretical insight on MBDLA can be gained through analytical techniques recently developed for cellular automata (see [15] and references therein). These reasons make us confident that MBDLA is a promising model to achieve a deeper knowledge of the connection between aggregation models and experiments. We hope this paper stimulates further theoretical and experimental work to establish the validity limits of this model.

It is a pleasure to thank I. Cirac for valuable discussions. A.S. is partially supported by DGICyT (Spain) through Grant No. PB92-0248 and by MEC/Fulbright. M.J.B. and J.M.R. acknowledge support from DGICyT (Spain) Grant No. MAT91-0031. Work at Los Alamos is performed under the auspices of the U.S. D.O.E.
[1] T. Vicsek, Fractal Growth Phenomena, 2nd ed. (World Scientific, Singapore, 1993).

[2] Dynamics of Fractal Surfaces, edited by F. Family and T. Vicsek (World Scientific, Singapore, 1991).

[3] T. A. Witten and L. M. Sander, Phys. Rev. Lett. 47, 1400 (1981); Phys. Rev. B 27, 5686 (1983).

[4] L. Niemeyer, L. Pietronero, and H. J. Wiesmann, Phys. Rev. Lett. 52, 1033 (1984).

[5] A. Sánchez, F. Guinea, L. M. Sander, V. Hakim, and E. Louis, Phys. Rev. E 48, 1296 (1993).

[6] J. Erlebacher, P. C. Searson, and K. Sieradzki, Phys. Rev. Lett. 71, 3311 (1993).

[7] J. M. Riveiro and M. J. Bernal, J. Non-Cryst. Solids 160, 18 (1993).

[8] G. S. Cargill and R. W. Cochrane, J. Phys. (Paris) Colloq. 35, C4-269 (1974); G. Dieta, H. Bestgen, and G. Goodlewsky, J. Magn. Magn. Mater. 13, 71 (1979); J. M. Riveiro and G. Rivero, IEEE Trans. Magn. MAG-17, 3082 (1981).

[9] T. Nagatani and F. Sagués, Phys. Rev. A 44, 6723 (1991); 44, 8303 (1991); T. Nagatani, Phys. Rev. 46, 2022 (1992).
[10] R. F. Voss, Phys. Rev. B 30, 334 (1984); J. Stat. Phys. 36, 861 (1984).

[11] Determined by chemical procedures by the vanado-molybdato method, see D. C. Abbott, G. E. Emsden, and J. Harris, Analyst 88, 814 (1963).

[12] A. Brenner, Electrodeposition of Alloys (Academic Press, New York, 1963), pp. 78ff, 468ff.

[13] New walkers were created above the top coordinate of the growing aggregate. This constraint was removed in several simulations to check whether three-dimensional effects could give rise to different results. It was then possible that walkers were created in closed empty regions of the aggregate. The outcome of the simulations was very much similar to those carried out with the constraint.

[14] One can also think of choosing different $s$ for Co and P ions. We did so and checked that the results changed very little even taking rather different values.

[15] J. M. Molera, F. C. Martínez, J. A. Cuesta, and R. Brito (unpublished). 


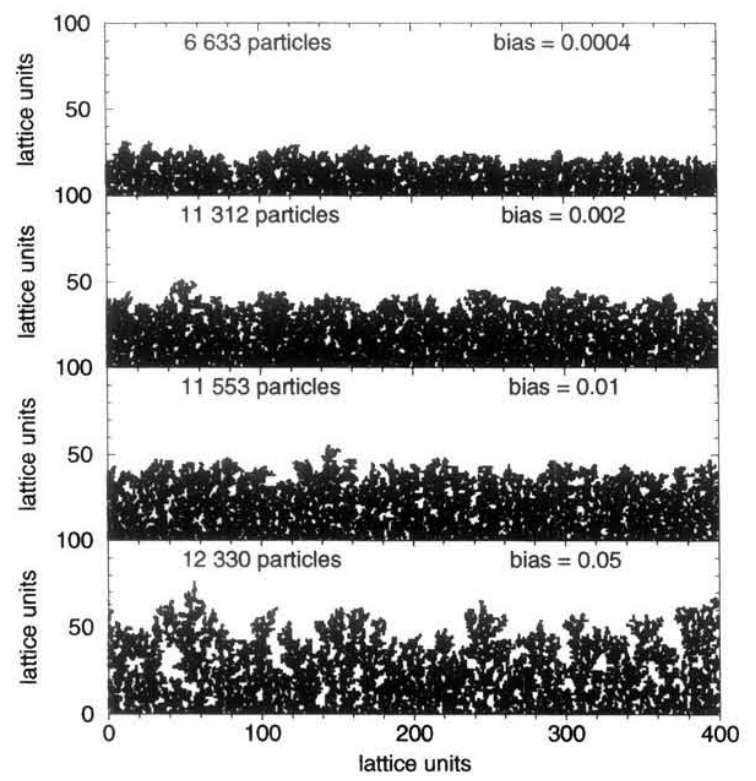

FIG. 2. Clusters obtained for different currents and times. Bottom to top: $J=2.5,0.5,0.1$, and $0.02 \times 10^{5} \mathrm{~A} \mathrm{~m}^{-2}$ (equivalent $p=0.05,0.01,0.002$, and 0.0004$)$, whereas $T_{M}=2,10,50$, and 60 min (equivalent $T_{\text {sim }}=2.8,14,70$, and $84 \times 10^{7}$ time steps). Note that the morphology becomes more open when increasing the current, becoming quite planar for the smallest $p$ value. The top aggregate was grown with $s$ four times the one it should have according to the linear relationship, to make it visible here. With the proper $s$ value $T_{M}$ would be $300 \mathrm{~min}$ (equivalent to $350 \times 10^{7}$ time steps). 